\title{
A Real Time Approach: ECG Noise Reduction in Chebyshev Type II Digital Filter
}

\author{
Sonal K. Jagtap \\ Research Student, Dept. of Electronics, Shivaji \\ University, Kolhapur (MS), India-416004
}

\author{
M. D. Uplane \\ Prof and Head, Dept. of Electronics, Shivaji \\ University, Kolhapur (MS), India-416004
}

\begin{abstract}
It is well known that biomedical signals carry important information about the behavior of the living systems under study. With the analysis of the Electrocardiogram (ECG) signal it may be possible to predict heart problems or monitor patient recovery after a heart intervention. A proper processing of these signals enhances their physiological and clinical information. The quality of biomedical signal is degraded mainly by many sources of noise such as power line interference (PLI), baseline drift, muscle contraction noise etc. The objective of this paper is to implement the Chebyshev Type II digital filter to overcome degradation by improving ECG signal quality for quality clinical diagnosis. Different artifacts are the reason in corruption of the ECG signal. Removing noise from the biomedical signal is still challenging and a rapidly expanding field with a wide range of applications in ECG noise reduction. Present paper deals with the design of Chebyshev Type II filters including low pass, high pass, notch filters. Also the performance is tested using cascading of filters. For real time application 1711 add on card is used. Different ECG signals from MIT/BIH arrhythmia database are used for validation and compared with real time ECG signals. The objective of this paper is to implement the Chebyshev Type II digital filter to overcome degradation by improving ECG signal quality for quality clinical diagnosis. Finally results indicate noise reduction in the ECG.
\end{abstract}

\section{Keywords}

Biomedical Signal, ECG, PLI, Chebyshev Type II, Digital Filter.

\section{INTRODUCTION}

ECG is a method to measure and record different electrical potentials of the heart. Owing to intensifying importance of biomedical signal processing, increasing efforts are devoted to noise reduction of biomedical ECG signal. The task can become complicated when quality is degraded due to interferences in ECG signal, making interpretation quite difficult. So removal of this noise is needed in ECG analysis for correct diagnosis. The ECG signal is typically in the range of $2 \mathrm{mV}$ and requires a recording bandwidth of 0.05 to 100 Hz. Under normal conditions, ECG tracings have a very predictable direction, duration, and amplitude. Because of this, the various components of the ECG tracing can be identified, assessed, and interpreted as to normal or abnormal function. The ECG is also used to monitor the heart's response to the therapeutic interventions. Because the ECG is such a useful tool in the clinical setting, the respiratory care practitioner must have a basic and appropriate understanding of ECG analysis. Fig. 1 shows the basic ECG waveform with schematic representation of a single cycle of ECG corresponding to one heart beat.

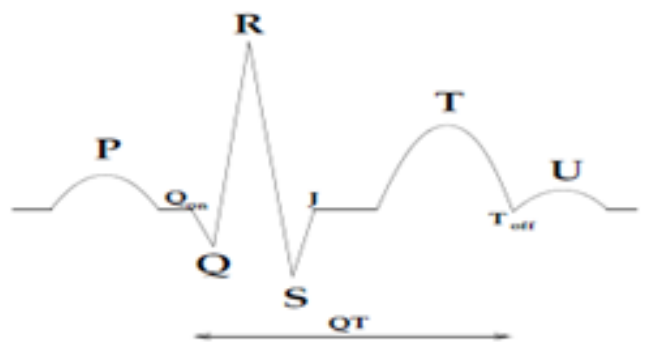

Fig 1: Schematic representation of a single cycle of ECG corresponding to one heart beat.

The signal is normally corrupted with two major noises generated by biological and environmental resources. The first group includes muscle contraction or electromyographic (EMG) interface, baseline drift, ECG amplitude modulation due to respiration and motion artifacts caused by changes in electrode skin impedance with electrode motion. The second group includes power line interference, electrode contact noise, instrumentation noise generated by electronic devices used in signal processing, electrosurgical noise and radio frequency. Different types of interferences are listed in fig.2.

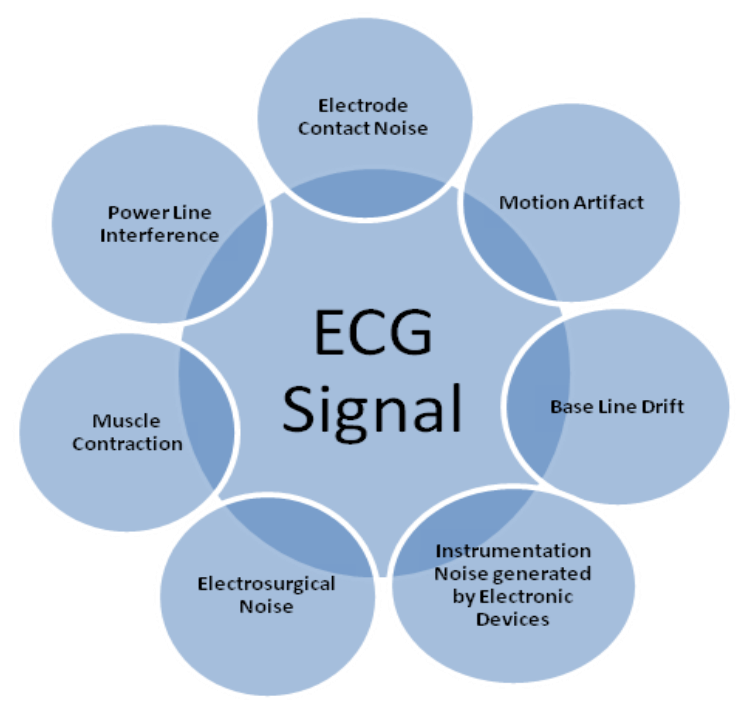

Fig 2: Interferences in the ECG signal

Hence several techniques have been presented in the literature to effectively reduce the noise in ECG analysis. 
Ferdjallah M. and Barr R. E. introduced frequency domain digital filtering techniques for removal of PLI [1]. Sornmo L. have applied time varying filtering techniques to the problem of baseline shift [2]. McManus C.D., Neubert K.D. and Cramer E. have compared digital filtering methods for elimination of AC noise in ECG [3]. Patrica Arand patented method and apparatus for removing baseline wander from an ECG signal [4]. Pei S.C., Tseng C.C proposed IIR notch filter with transient suppression in ECG [5]. Hamid Gholam, Hosseini, Homer Nazeran, Karen J. Reynolds elaborated on ECG noise cancellation using application of digital filter [6]., A nonlinear adaptive method of elimination of powerline interference in ECG signals was developed by Ziarani a. K. and Konard A. [7]. Mitov I.P. A method for reduction of power line interference in the ECG [8]. Yong Lian, Poh Choo Ho. Focued on multiplier free digital filter [9]. Lisette P. Harting, Nikolay M. Fedotov, Cornelis H. Slump were discussed on baseline drift suppression in ECG recording [10]. Dotsinky I., Stayanov T. discussed on power-line interference cancellation in ECG signals [11]. Jacek M. Leski, Norbert Hezel have proposed a combination of ECG baseline wander and PLI reduction using nonlinear filter band [12]. Lu G.et al. have suggested a fast convergence of recursive least square algorithm to enable the filter to track complex dystonic EMGs and to effectively remove ECG noise. The adaptive filter procedure proved a reliable and efficient tool to remove ECG artifact from surface EMGs with mixed and varied patterns of transient, short and long lasting dystonic contractions [13]. A new asynchronous averaging and filtering (AAF) algorithm is proposed by Gautam A. et al. for ECG signal denoising. AAF algorithm reduces random noise (major component of EMG noise) from an ECG signal and provides comparatively good results for baseline wander noise cancellation. SNR improves in a filtered ECG signal, while signal shape remains undistorted. An AAF algorithm is more advantageous than adaptation algorithms like Wiener and LMS algorithm [14]. Sorensen J. S.et al. have described a comparison of IIR and wavelet filtering for noise reduction of the ECG. Ideally, the output of the optimal filter has perfect noise removal, no distortion and low computation time. This criterion was used to select one wavelet filter and one IIR filter to be used on the ECG with transient muscle activity. For this signal the root mean square error (RMSE) of the non noise and noise segment were calculated using the selected wavelet and IIR filters [15]. Gupta R., Bera J.N. and Mitra M. have developed a simple, cost effective online ECG acquisition system for further data processing. An 8051 based dedicated embedded system is used for converting the digitized ECG into serial data, which is delivered to a standalone PC through a 'COM' port for storage and analysis. A serial link is preferred as it minimizes cable costs and interference effects as is the case with a parallel link. The developed MATLAB based graphical user interface (GUI) facilitates a user to control the operations on the entire system [16]. Luo S. and Jhonston P. have discussed the issues related to the inaccuracy of ECG preprocessing filters. Their investigations are in the context of facilitating efficient ECG interpretation and diagnosis as a review [17].

In this paper, Chebyshev Type II filter is designed and implemented. Finally improvement in ECG signal by noise reduction is presented and discussed. This paper aims towards simulink based design of digital filters which further can be extended to interface with the processor.

\section{METHODOLOGY}

This filter is named in honor of Pafnuty Chebyshev (Chebyshov, Tchebycheff or Tschebyscheff) a great Russian mathematician because their mathematical characteristics are derived from chebyshev polynomials. Chebyshev filters are analog or digital filters having a steeper roll off and more pass band ripple than Butterworth filters. Chebyshev filters have the property that they minimize the errors between the idealized filter characteristic and actual over the range of filter. There are two types of chebyshev filters as follows.

- Chebyshev type I.

- Chebyshev type II.

\subsection{Chebyshev Type I filter}

The chebyshev low pass filter has a magnitude response given by

$$
\| H(j \omega)\rangle=\frac{A}{\left[1+\varepsilon^{2} C_{w}^{2}\left(\frac{\Omega}{\Omega_{C}}\right)\right]^{\frac{1}{2}}}
$$

where $\mathrm{A}$ is the filter gain, $\varepsilon$ is a constant and $\Omega_{e}$ is the $3 \mathrm{~dB}$ cut off frequency.

The chebyshev polynomial of the first kind of $\mathrm{N}^{\text {th }}$ order, $C_{N}(x)$ is given by

$$
C_{M}(x)=\left\{\begin{array}{l}
\cos \left(N \cos ^{-1} x\right) \text {. for }\|x\| \leq 1 \\
\cos \left(N \cosh ^{-1} x\right) . \text { for }\|x\| \geq 1
\end{array}\right.
$$

The magnitude response of the chebyshev filter is shown in fig. 3. It has equiripple pass band and maximally flat stop band. It is further seen that with increase in filter order, the chebyshev response approximates the ideal response. The phase response of the chebyshev filter is more nonlinear than the butterworth filter for a given filter of length $\mathrm{N}$.

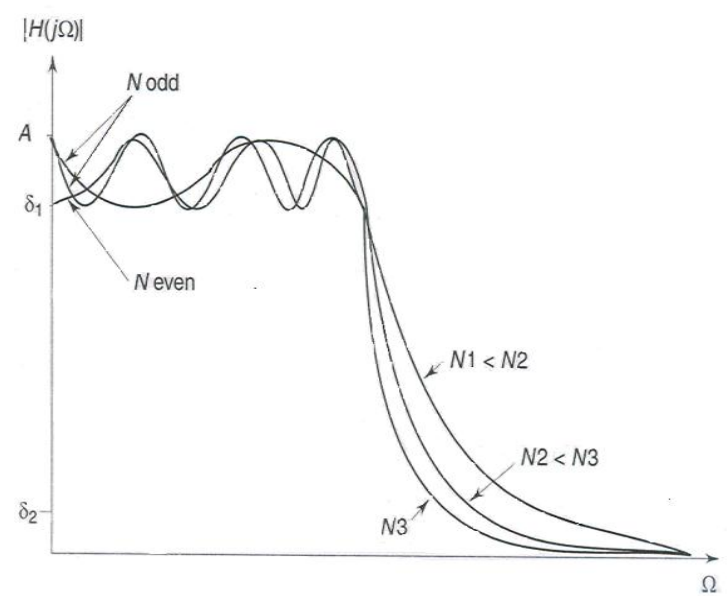

Fig 3: Magnitude response of a low pass Chebyshev Type I filter

\subsection{Chebyshev Type II filter}

Inverse Chebyshev filters are also called Type II Chebyshev filters. The inverse chebyshev low pass filter has a magnitude response given by 


$$
|H(j \Omega)|=\frac{a C_{M}\left(\frac{\Omega_{2}}{\Omega_{e}}\right)}{\left[1+\varepsilon^{2} C_{M}^{2}\left(\frac{\Omega_{2}}{\Omega}\right)\right]^{\frac{1}{2}}}
$$

where $\varepsilon$ is a constant and $\Omega_{c}$ is the $3 \mathrm{~dB}$ cut off frequency. The chebyshev polynomial $C_{N}(x)$ is given by equation (2).

The magnitude response of the chebyshev type II filter is shown in fig. 4. It has maximally flat pass band and equiripple stop band, just the opposite of the Chebyshev type I filter response. That is why the Chebyshev Type II filters are called the inverse Chebyshev filters.

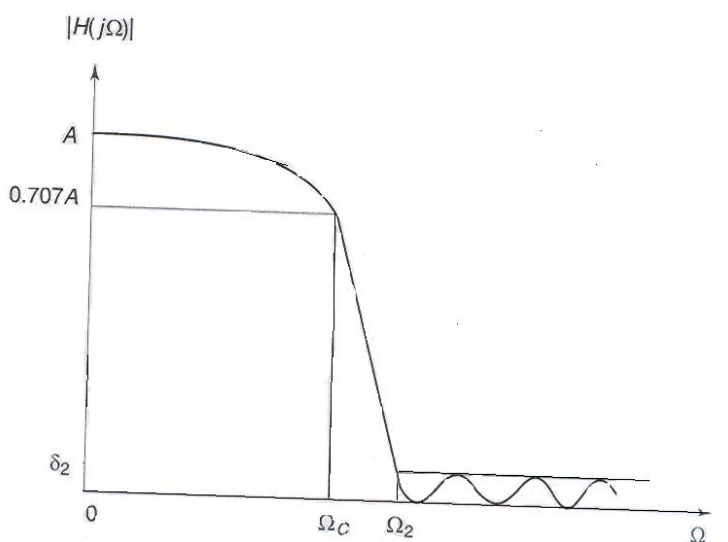

Fig 4: Magnitude response of a low pass Chebyshev Type II filter

\section{PERFORMANCE ANALYSIS}

In this paper performance analysis has been carried out with the help of Chebyshev Type II filter.

\subsection{Chebyshev Filter Application}

Recording the electrical activity of the heart is nothing but first step in the ECG analysis. This can be done noninvasively with the help of electrodes mounted on the surface of the body. Here we are using ECG amplifier interface for real time data acquisition card i.e. PCI 1711. The filters are designed using MATLAB FDA Tool by specifying the filter order, cut off frequency and sampling frequency. In the present section, the design considerations of low pass filter, high pass filter, notch filter are elaborated separately for removal of noise signals from ECG using Chebyshev Type II filter. The proposed block diagram of Chebyshev Type II filter is shown in fig. 5 .

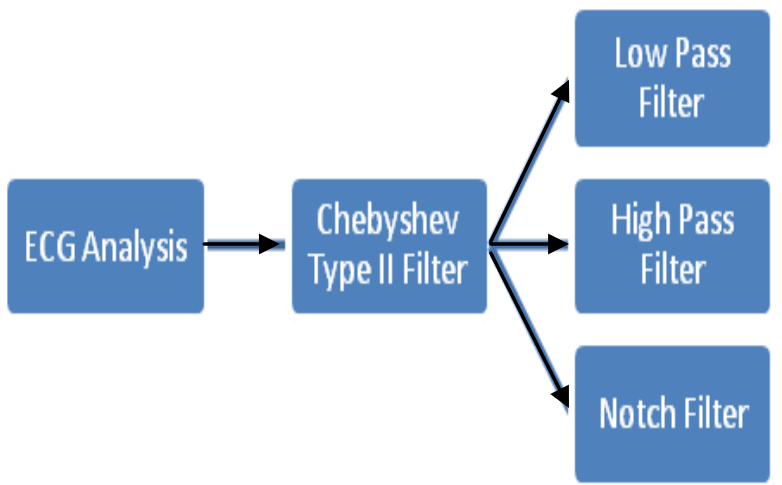

Fig 5: Proposed block diagram of Chebyshev filter

\subsubsection{Design of Chebyshev Type II Low Pass}

Filter: Removal of High Frequency Noise

The information is present in frequency range of $0.5 \mathrm{~Hz}$ to 100 $\mathrm{Hz}$ is very useful. All the signals above $100 \mathrm{~Hz}$ is treated as noise. The section deals with design and implementation of the low pass filter were designed for sampling frequency 1000 $\mathrm{Hz}$ and filter order 5 for the removal of high frequency noise in ECG signal. Simulation model for implementation of low pass filter is shown in fig. 6 . Results for the same explained in section 4.1.

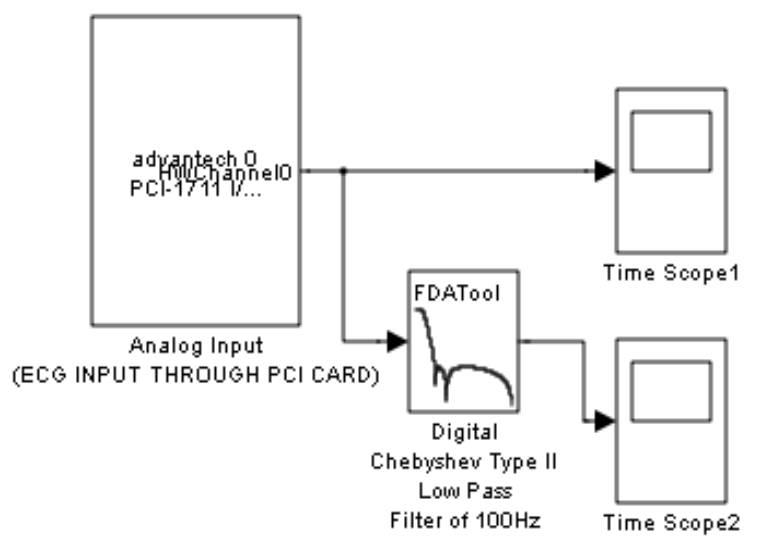

Fig 6: Simulation model for implementation of low pass filter

\subsubsection{Design of Chebyshev Type II High Pass}

Filter: Removal of Low Frequency Noise

In the present design of high pass filter, the order of the filter is considered is 3 . Though the order of filter is less it gives satisfactory results. Baseline wander is minimised by design of high pass filter is focused in this section. Chebyshev Type II high pass filter was designed with filter order 3 and sampling frequency $1000 \mathrm{~Hz}$. Simulation model for implementation of high pass filter is shown in fig. 7. Results for the same explained in section 4.2.

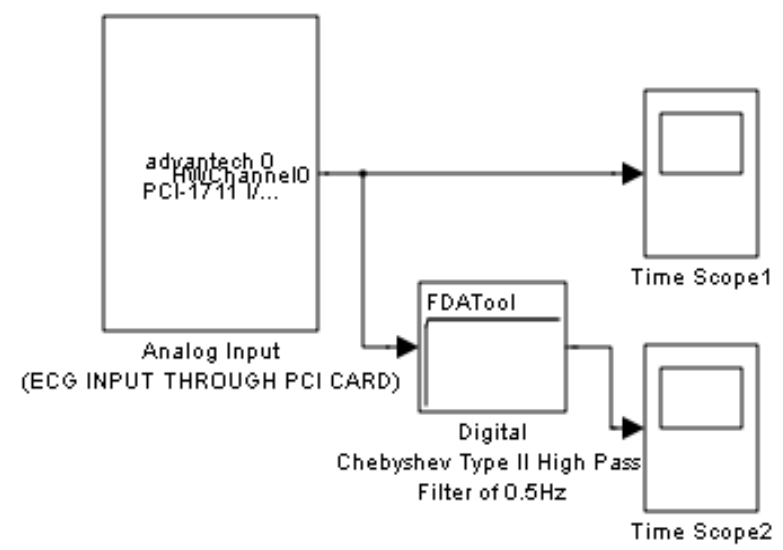

Fig 7: Simulation model for implementation of high pass filter

\subsubsection{Design of Chebyshev Notch Filter: Removal} of Power Line Interference

Notch filter is implemented to avoid the problem of PLI in the ECG signal. In the present section the Butterworth notch filter has been designed for order 4 and sampling frequency 1000 $\mathrm{Hz}$ and implemented on the ECG signal containing power line interference. Simulation model for implementation of high 
pass filter is shown in fig. 8. Results for the same explained in section 4.3.

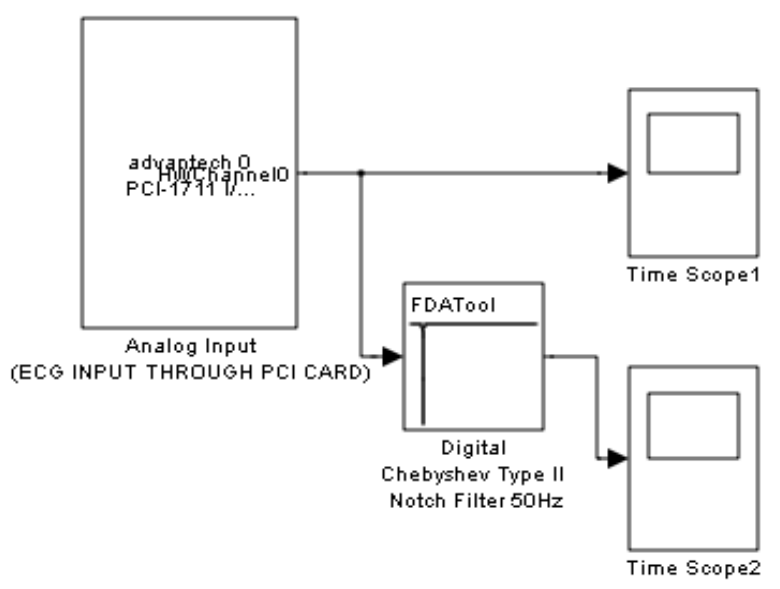

Fig 8: Simulation model for implementation of notch filter

\section{EXPERIMENTATION}

Individual experimentation on low pass, high pass, notch filter is carried out separately and signal power before and after filtration is discussed in this section.

\subsection{Implementation of Chebyshev Type II}

\section{Low Pass Filter}

Fig. 6 shows simulation model for low pass filtration. After implementation, fig. 9 shows ECG signal before and fig. 10 shows signal after low pass filtration respectively.

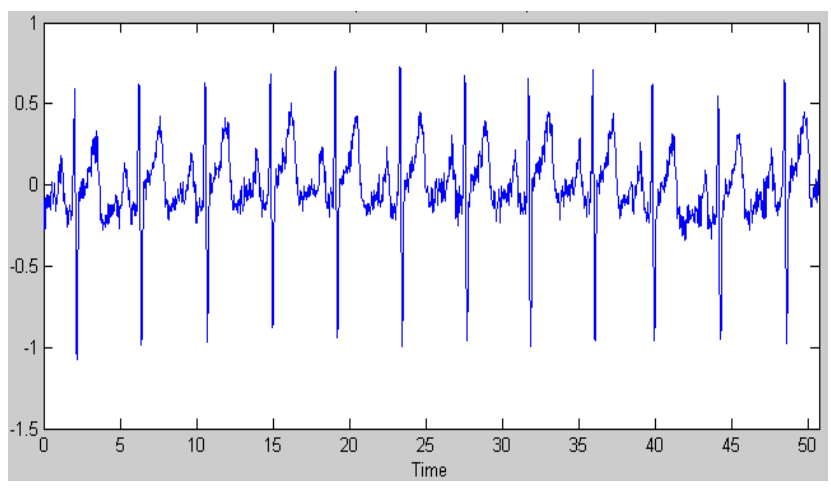

Fig 9: ECG signal before low pass filtration

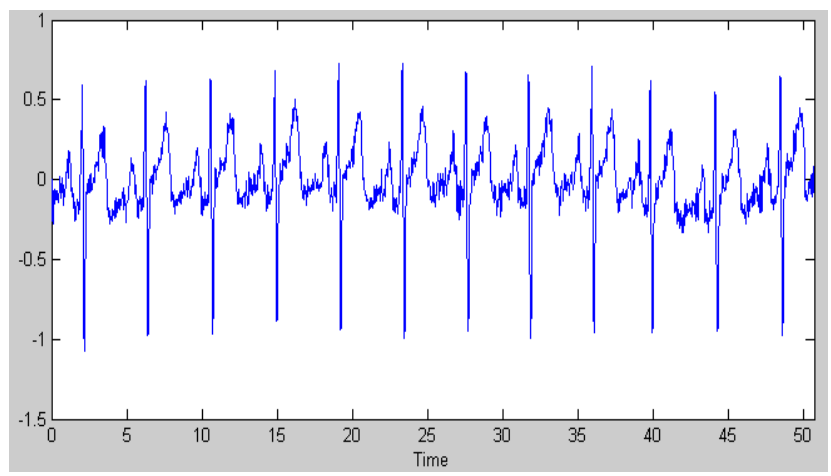

Fig 10: ECG signal after low pass filtration

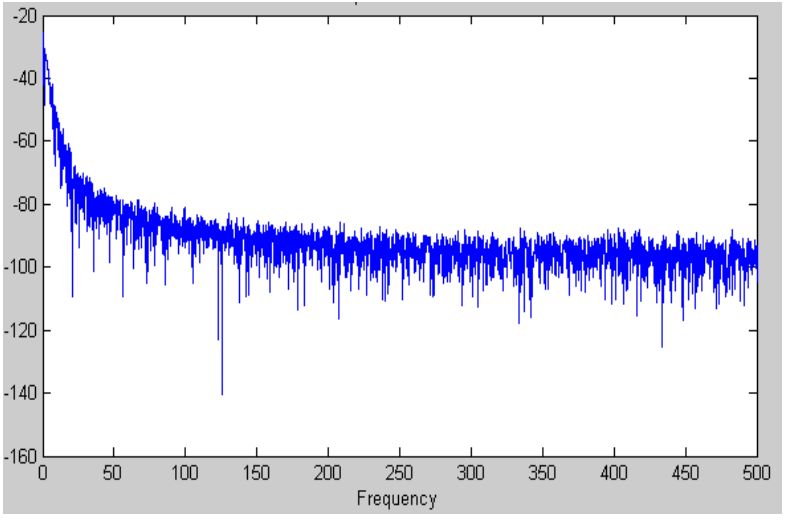

Fig 11: Frequency spectrum before low pass filtration

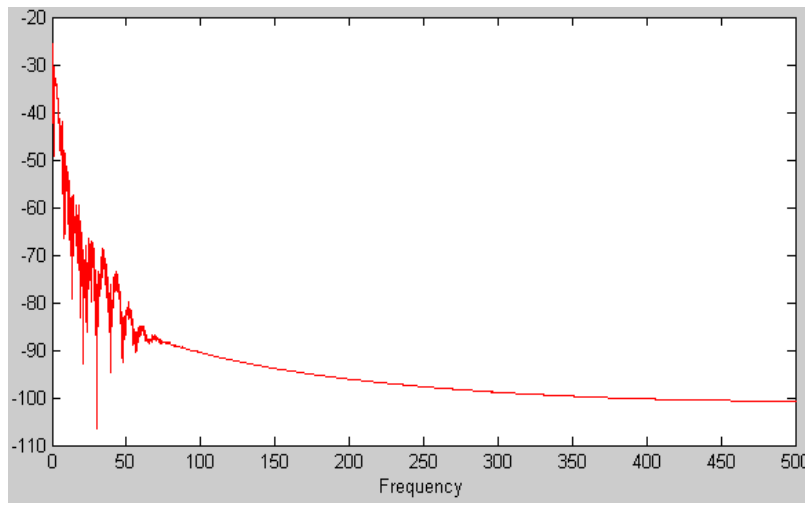

Fig 12: Frequency spectrum after low pass filtration

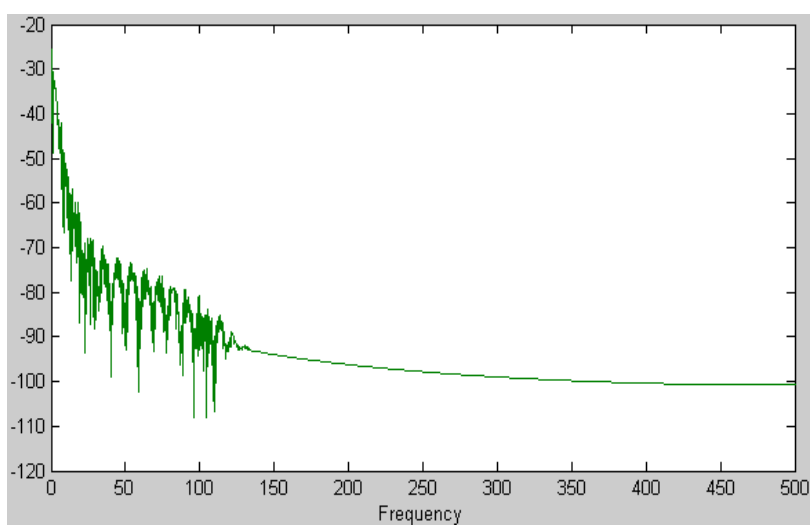

Fig. 13: Frequency spectrum with minimum order after low pass filtration

At a frequency of $100 \mathrm{~Hz}$, before filtration frequency spectrum exhibits signal power of $-105 \mathrm{~dB}$ and average power is higher i.e.- $125 \mathrm{~dB}$. After application of filter, power dropped to -95 $\mathrm{dB}$ shows removal of high frequency noise. Fig. 9 shows power spectrum before \& fig. 11, 12 shows after low pass filtration.

\subsection{Implementation of Chebyshev Type II High Pass Filter}

Fig. 7 shows simulation model for high pass filtration. After implementation, fig. 14 shows ECG signal before and fig. 15 shows signal after high pass filtration respectively. 


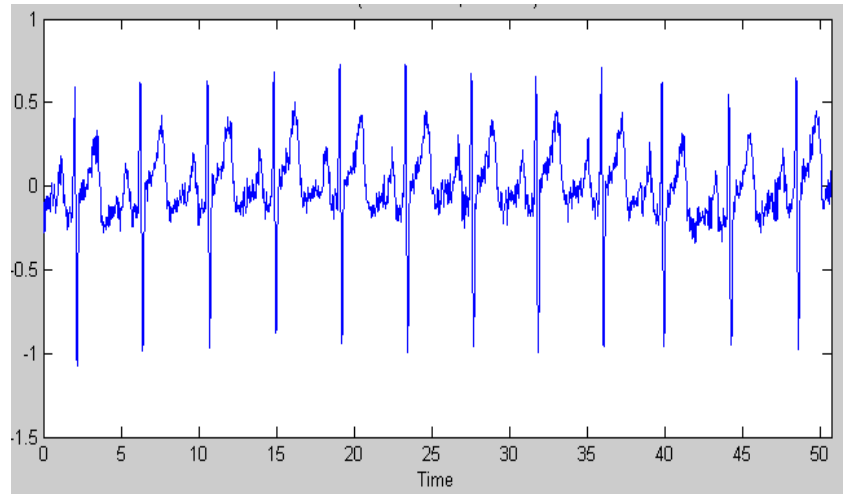

Fig 14: ECG signal before high pass filtration

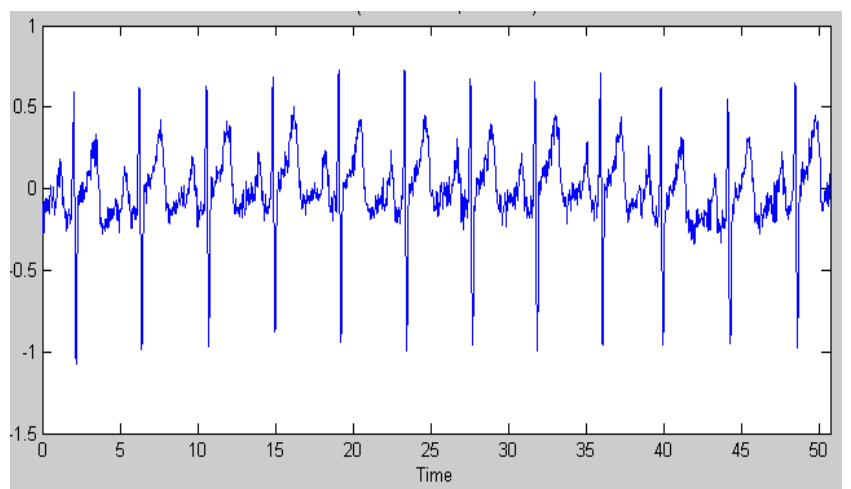

Fig 15: ECG signal after high pass filtration

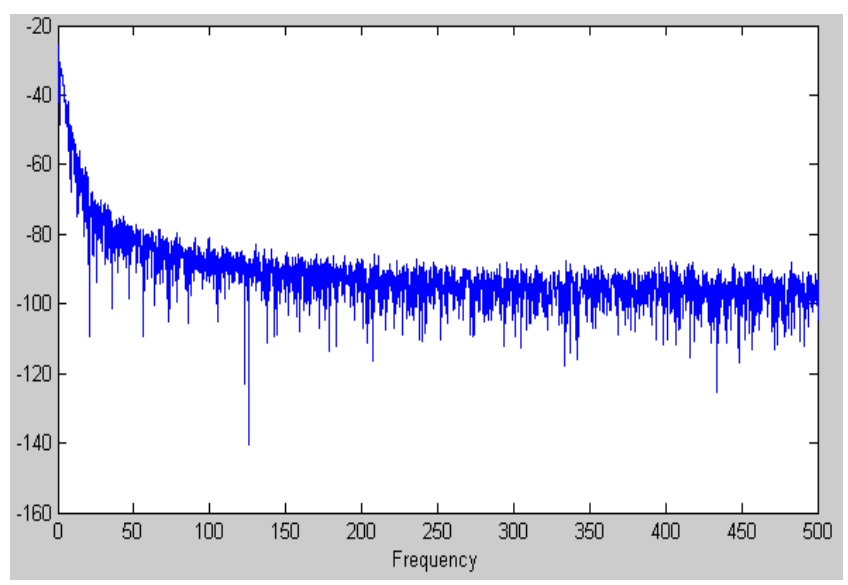

Fig 16: Frequency spectrum before high pass filtration

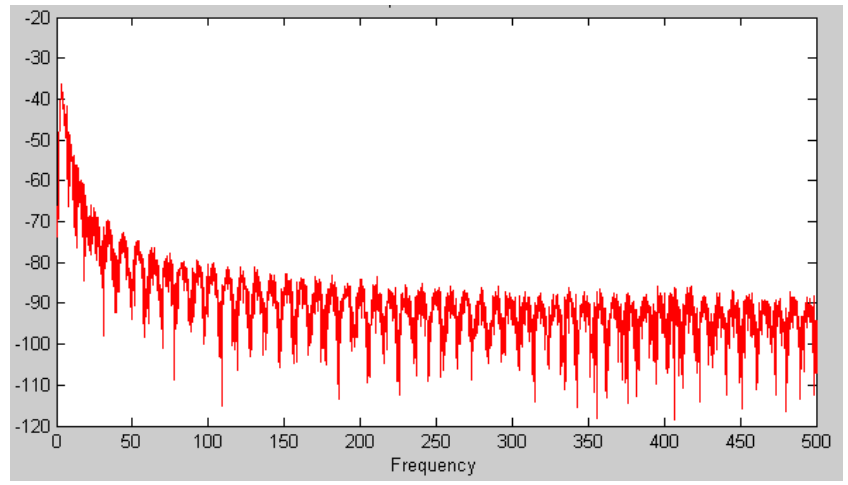

Fig 17: Frequency spectrum after high pass filtration

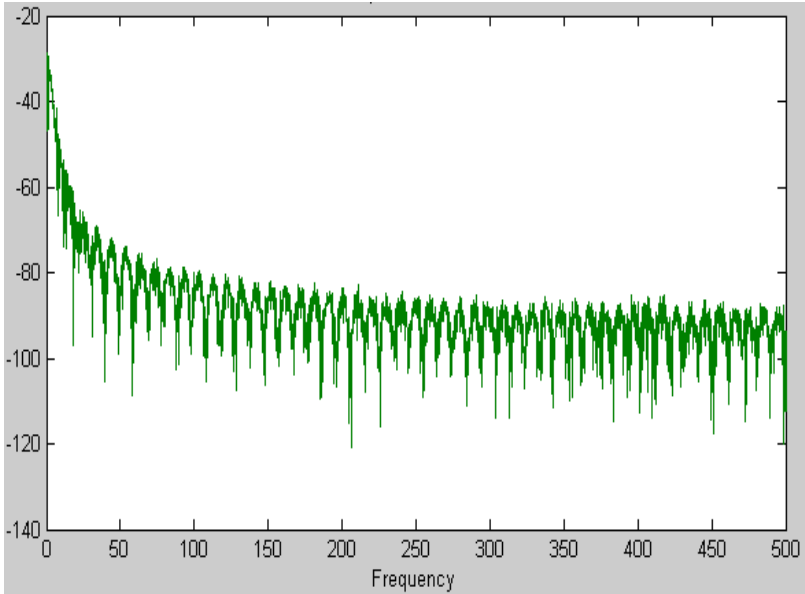

Fig 18: Frequency spectrum with minimum order after high pass filtration

Before filtration the signal power varies from $-30 \mathrm{~dB}$ to the $-50 \mathrm{~dB}$. It has been seen that power is reduced to the $-35 \mathrm{~dB}$ to $-75 \mathrm{~dB}$ indicating removal of low frequency signals.

\subsection{Implementation of Chebyshev Type II Notch Filter}

At a frequency of $100 \mathrm{~Hz}$ fig. 8 shows simulation model for Chebyshev type II notch filtration. After implementation, fig. 19 shows ECG signal before and fig. 20 shows signal after Chebyshev type II notch filtration respectively.

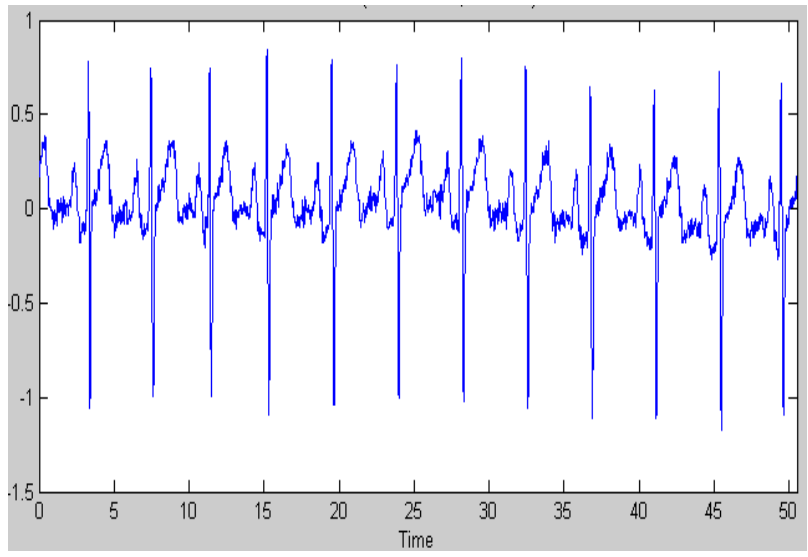

Fig 19: ECG signal before notch filtration

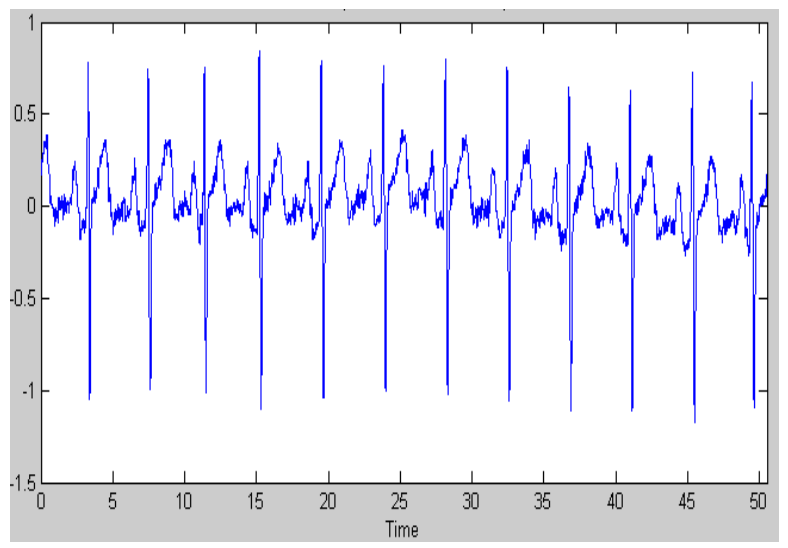

Fig 20: ECG signal after notch filtration 


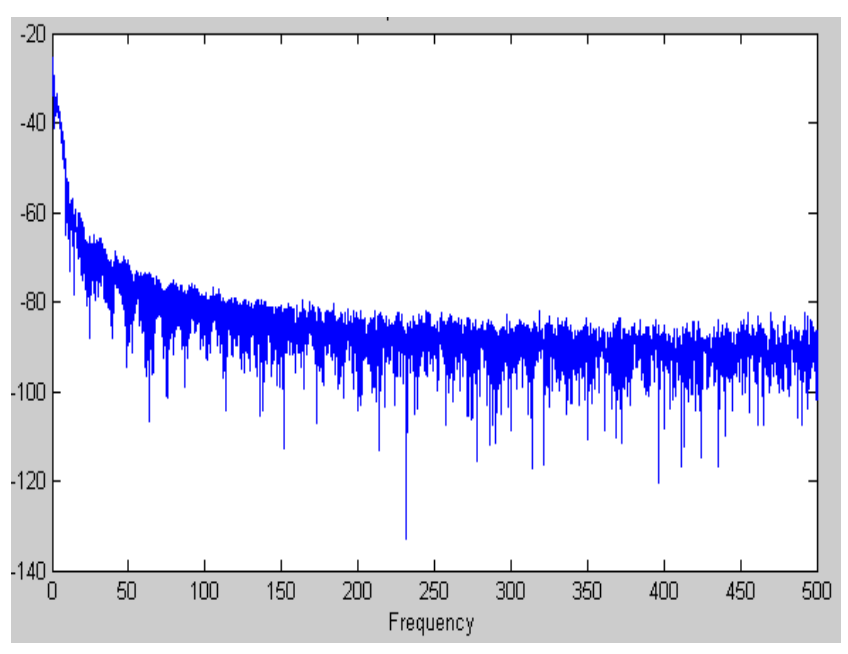

Fig 21: Frequency spectrum before application of notch

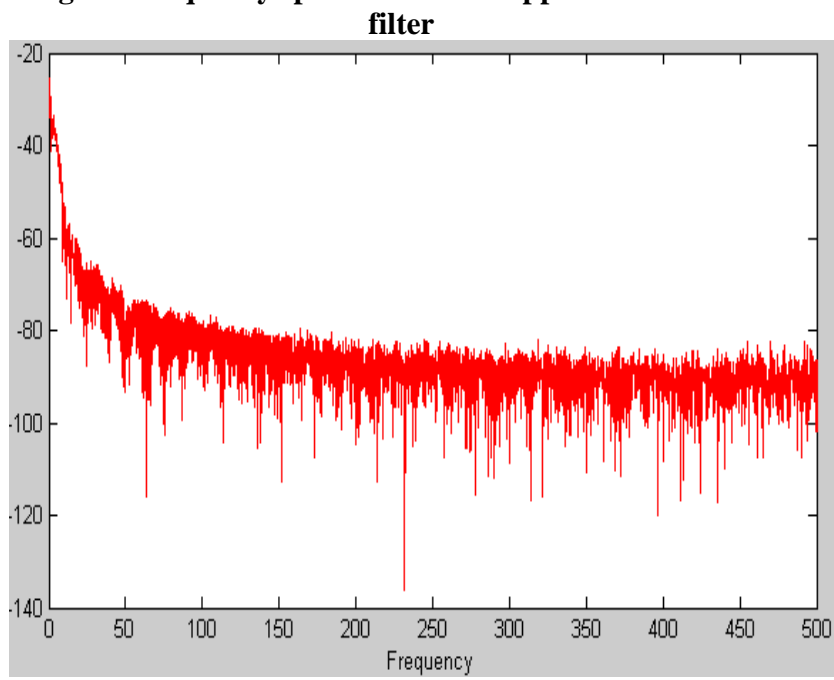

Fig 22: Frequency spectrum after application of notch filter

Fig.19 shows frequency spectrum of ECG signal before filtration it is seen that power of the signal is equivalent to the $50 \mathrm{~Hz}$ is $-90 \mathrm{~dB}$. From fig. 20, also when it is passed through notch filter power is reduced to $-110 \mathrm{~dB}$. From the figure it is observed that power line interference is reduced.

\section{RESULTS}

\subsection{Cascading of Chebyshev Type II Filter}

Finally, the filters are cascaded to achieve the combined effect of these filters. Final model is built in SIMULINK to remove the noise from the ECG. Final model includes Chebyshev Type II low pass filter, Chebyshev type II high pass filter and a notch filter centered at $50 \mathrm{~Hz}$. Individual performance as well as performance of cascade combination of filters has been compared.

Different ECG signals from MIT/BIH arrhythmia database are used for validation and compared with these real time ECG signals. The same results has been carried out for 4096 samples using same filters i.e. low pass, high pass and notch by considering text input as one of the ECG signal. Whatever results discussed here are limited upto $1000 \mathrm{~Hz}$ sampling frequency. Fig. 26 shows model used for cascading of Chebyshev Type II filter. It has been observed that this method of filtering focuses on maximum suppression of baseline wander with distortions in ST part of the segment.

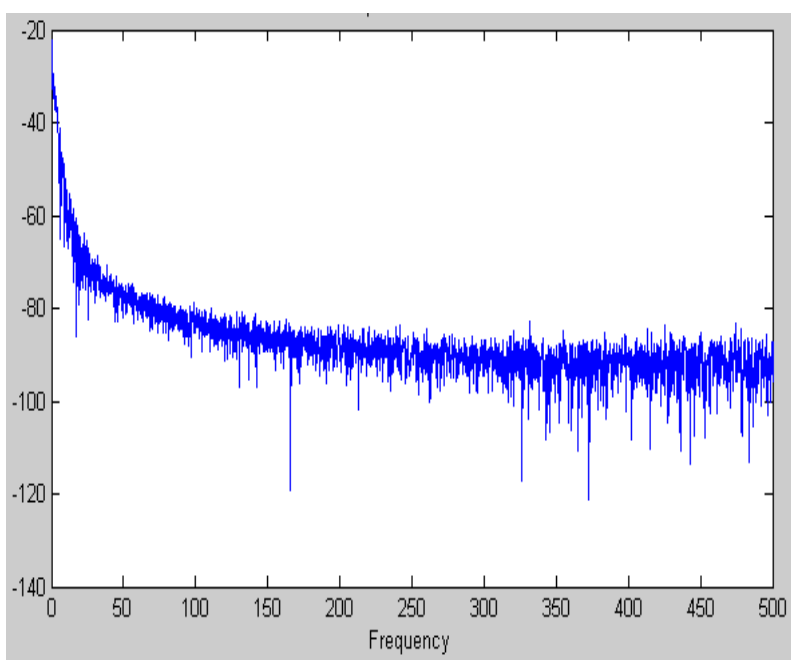

Fig 23: Frequency spectrum before application of cascade combination of filters

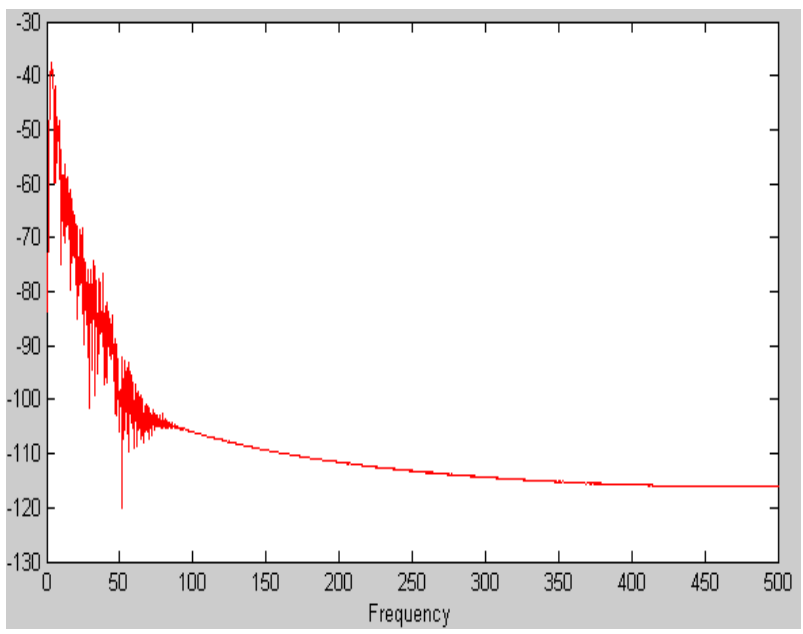

Fig 24: Frequency spectrum after application of cascade combination of filters

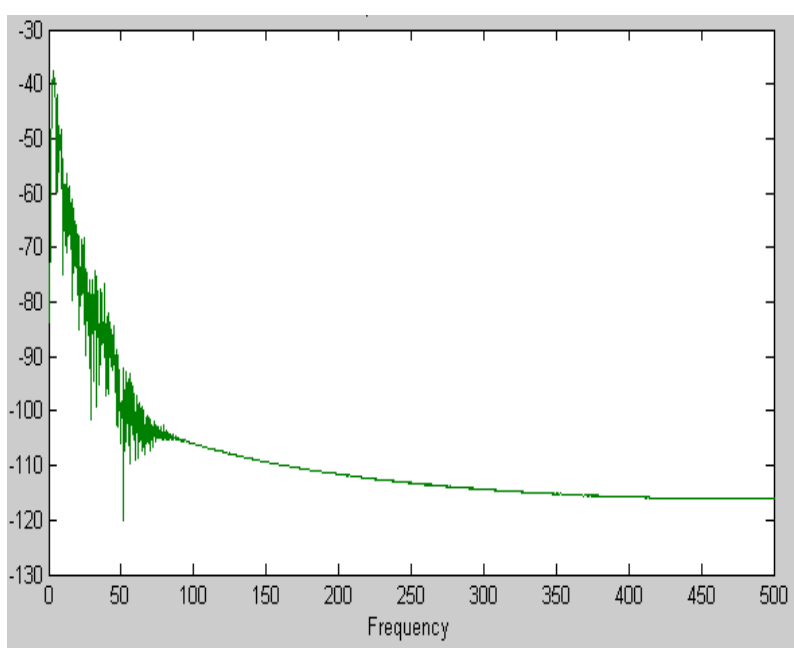

Fig 25: Frequency spectrum with minimum order after application of cascade combination of filters 


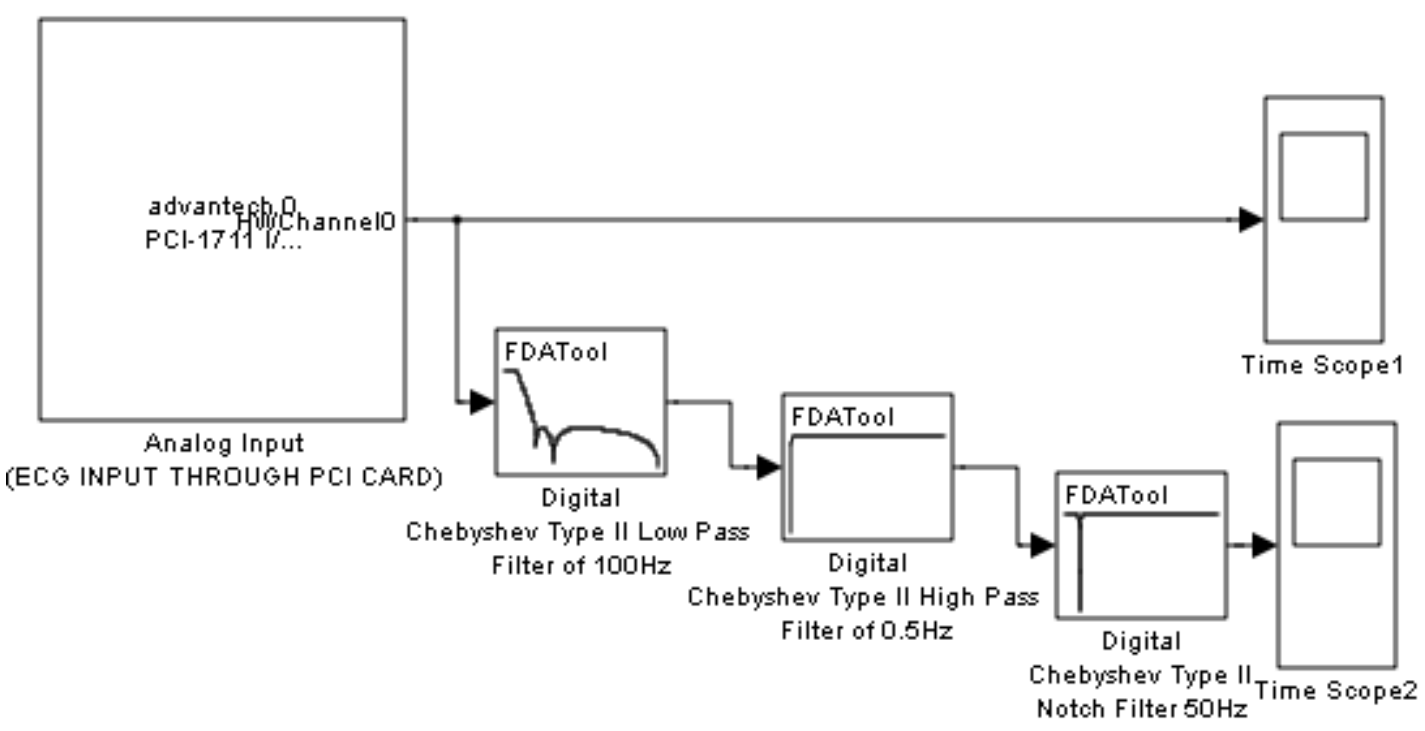

Fig 26: Model used for cascading of Chebyshev type II filter

\section{CONCLUSIONS}

Digital filter is the preeminent solution that caters the noise reduction up to satisfactory level. A digital Filter technique is best suited for ECG analysis and thereby helps in improving the quality of ECG signal with the help of Chebyshev Type II filter. From the results, it is seen that the filters reduces the low and high frequency components. The power line noise is also reduced. It is seen tip of the QRS complex is distorted. The outputs of the Chebyshev II filter shows that after using filter there is distortion in the ST part of the waveform. Both the filters work Satisfactory. Further this work can be applied on different types of filters and then depending on performance final model can be implemented to estimate the better quality signal.

\section{FUTURE SCOPE}

Yet a new path has been opened and only the future will be able to judge if this method can be successfully applied with the cascading of IIR and FIR filters for real time ECG systems. Finally, it is seen that an optimum combination can be implemented using FPGA technique.

\section{ACKNOWLEDGMENTS}

The Author would like to express sincere thanks to the Department of Electronics, Shivaji university, Kolhapur as well as Founder President of Sinhgad Technical Education Society Hon. Prof. M.N. Navale as well as for encouragement and timely support in accomplishment of the work. Also this work is supported by BCUD, University of Pune under research grant scheme. The author is grateful for this financial support.

\section{REFERENCES}

[1] Ferdjallah M., Barr R.E., "Frequency domain digital filtering techniques for the removal of powerline noise with application to the electrocardiogram", Comput. Biomed Res. 1990 Oct., 23(5), 473-489.

[2] Sornmo L., "Time-varying digital filtering of ECG baseline wanders", Med. Biol Eng Comput., 1993 Sept., 31(5), 503-508.
[3] McManus C D, Neubert K D,Cramer E, "Characterization and elimination of AC noise in electrocardiograms : a comparison of digital filtering methods" Comput. Biomed Res., 1993 Feb., 26(1), 4867.

[4] Patrica Arand, "Method and apparatus for removing baseline wander from an ECG signal", US patent Issued on June 7, 1994.

[5] Pei S.C., Tseng C.C., "Elimination of AC interference in electrocardiogram using IIR notch filter with transient suppression" IEEE Trans Biomed Eng., 1995 Nov, 42(11), 1128-1132.

[6] Hamid Gholam,Hosseini, Homer Nazeran, Karen J. Reynolds, "ECG noise cancellation in using digital filter", $2^{\text {nd }}$ international Conference on biomagnetism, 1998 Feb, Melbourne, Australia, pp.151-152.

[7] Ziarani a. K., Konard A., "A nonlinear adaptive method of elimination of powerline interference in ECG signals", IEEE Trans Biomed Eng., 2002 Jun, 49(6), 540-547

[8] Mitov I.P., "A method for reduction of power line interference in the ECG" ,Med Eng Phys., 2004 Dec., 26(10), 879-887.

[9] Yong Lian, Poh Choo Ho., "ECG noise reduction using multiplier free digital filter" ,ICSP`04 proceedings IEEE conference 2004, 2198-2201.

[10] Lisette P. Harting, Nikolay M. Fedotov, Cornelis H. Slump, "On baseline drift suppression in ECG recording" ,Proceeding of IEEE Benelux Signal Processing Symposium 2004,pp. 133-136.

[11] Dotsinky I., Stayanov T., "Power-line interference cancellation in ECG signals" Biomed Instrum Technol. 2005 Mar,39(2),155-162.

[12] Jacek M. Leski, Norbert Hezel, “ ECG baseline wander and power line interference reduction using nonlinear filter band", Signal Processing, Elsevier, 85(2005), 781793 
[13] Lu G., Brittan J-S, Holland P., Yianni J., Green A.L., Stein J.F., Aziz T.Z., Wang S., "Removing ECG noise from surface EMG signals using adaptive filtering", Neuroscience letters. 2009, 462, 14-19.

[14] Gautam A., Lee H-J, Chung W.Y., "ECG signal denoising with asynchronous averaging and filtering algorithm", International Journal of Healthcare Information Systems and Informatics. 2010 April-Jun; 5(2), 30-36.

[15] Sorensen J.S., Johannesen L., Grove U.S.L, Lundhus K., Couderc J-P, Graff C., "A comparison of IIR and wavelet filtering for noise reduction of the ECG", Comput Cardiol. 2010 Sept. 26, 37, 489-492.

[16] Gupta R., Bera J.N., Mitra M., "Development of an embedded system and MATLAB based GUI for online acquisition and analysis of ECG signals", Measurement 2010, 43, 1119-1126.

[17] Luo S., Jhonston P., “A review of electrocardiogram filtering”, J. Electrocardiol. 2010, 43, 486-496.

[18]Database:www.physionet.org/physiobank/database/mitdb. 